\title{
Application of Virtual Surgical Planning with Computer Assisted Design and Manufacturing Technology to Cranio-Maxillofacial Surgery
}

\author{
Linping Zhao ${ }^{1,2,3}$, Pravin K. Patel ${ }^{1,2,3}$, Mimis Cohen $^{1,2}$ \\ ${ }^{1}$ The Craniofacial Center, University of Illinois Medical Center; ${ }^{2}$ Division of Plastic, Reconstructive and Cosmetic Surgery, Department of \\ Surgery, University of Illinois; ${ }^{3}$ Shriners Hospitals for Children-Chicago, Chicago, IL, USA
}

\begin{abstract}
Computer aided design and manufacturing (CAD/CAM) technology today is the standard in manufacturing industry. The application of the CAD/CAM technology, together with the emerging 3D medical images based virtual surgical planning (VSP) technology, to craniomaxillofacial reconstruction has been gaining increasing attention to reconstructive surgeons. This article illustrates the components, system and clinical management of the VSP and CAD/CAM technology including: data acquisition, virtual surgical and treatment planning, individual implant design and fabrication, and outcome assessment. It focuses primarily on the technical aspects of the VSP and CAD/CAM system to improve the predictability of the planning and outcome.
\end{abstract}

Keywords Computer aided design and manufacturing / Computer assisted image processing / Computer-assisted surgery / Reconstructive surgical procedures / Three-dimensional imaging

\author{
Correspondence: Linping Zhao \\ The Craniofacial Center, University \\ of Illinois Medical Center, 811 South \\ Paulina Street, Chicago, IL 60612, \\ USA \\ Tel: +1-312-996-9313 \\ Fax: +1-312-996-7546 \\ E-mail: linping.zhao@gmail.com
}

The VSP+CAD/CAM system presented is partially supported by Face the Future Foundation, and Shriners Hospitals for Children.

No potential conflict of interest relevant to this article was reported.

Received: 11 Mar 2012 • Revised: 27 Mar 2012 • Accepted: 28 Mar 2012

pISSN: 2234-6163 • elSSN: 2234-6171 • http://dx.doi.org/10.5999/aps.2012.39.4.309・ Arch Plast Surg 2012;39:309-316

\section{INTRODUCTION}

The benefits of increased productivity with predictable outcome became obvious in the automobile and aerospace industries in the 1970s as a result of the application of the computer-aided design $(\mathrm{CAD})$ and computer-aided manufacturing (CAM) technology. With industry the widespread use of CAD/CAM technology produced both the momentum and desire to translate three-dimensional (3D) images into physical prototype models. In parallel, computed tomography (CT) imaging captured for the first time the living human anatomy in 3D [1,2]. It then became possible to translate the $\mathrm{CAD} / \mathrm{CAM}$ technology to potential applications in the medical and dental fields.

The impact of the CAD/CAM technology, in particular, the rapid prototyping $(\mathrm{RP})$ technology, together with the available of the 3D medical images (CT and magnetic resonance imaging $[\mathrm{MRI}])$ and medical image analysis software, has been transforming clinical practice in craniomaxillofacial surgery in the past decades. Today the applications extend from the custom fabricated craniofacial prosthetic implants [3] to occlusal guides for orthognathic surgical procedures [4].

This article illustrates the components, system and clinical management of the virtual surgical planning (VSP) and CAD/ CAM technology including: data acquisition, virtual surgical and treatment planning, individual implant design and fabrication, and outcome assessment. It focuses primarily on the technical aspects of the VSP and CAD/CAM system to improve the predictability of the planning and outcome. 


\section{A BRIIEF HISTORICAL REVIEW}

The application in the field of craniomaxillofacial surgery can be traced back as early as the 1980's when the anatomical model with detailed geometric features were built from the CT scan slices $[5,6]$. Initially, this was achieved using computer numerically controlled ( $\mathrm{CNC}$ ) milling machines that used the $3 \mathrm{D}$ data to cut the shape of each CT 'slice' from a solid block of styrofoam or polyurethane. This late 1980's technology was used to produce rather crude physical models of heads and faces with 'stepped' surfaces reflecting both the 'sliced' CT data with the height or the slice thickness of 3 to $6 \mathrm{~mm}$ and the capacity of the milling machine at that time. With time this system evolved producing more accurate models [7-9] and simple prostheses [3,10,11]. However, the limitation to this method is that the level of complexity that is required for detailed anatomical models cannot be reproduced even by five-axis milling machines [12].

A more useful approach is the RP technology, such as selective laser sintering (SLS) and stereolithography (SLA) that emerged in late 1980s [13-15], and 3D printer that is popular today [4]. The RP technology works on the principle of building up the model in layers or slices by material deposition rather than cutting down a block of polyurethane. As an anatomical part can be scanned into a computer system slice by slice, similarly an object can be faithfully reproduced slice by slice using the $3 \mathrm{D}$ computer data in conjunction with a RP machine. As the model is created tomographically, it contains nearly all the details of its internal contour geometry, not just the outer surface as in the milling technique [16]. Using this technology, life size 3D solid skull models were made and used to select optimal bone graft donor sites $[17,18]$ or used as a guide to fabricate implants [19-21].

In addition to the RP technology, several critical technological developments in last two to three decades contributed to the widespread use of the CAD/CAM technology from the patient specific models to individualized implants, prosthesis, to the surgical panning and guidance. Among the developments were: 1) the improvement of image quality and resolution in medical images with CT scan capable of less than $1 \mathrm{~mm}$ in slice thickness, 2) Cone-beam CT scanners that provide maxillofacial information with finer resolution and considerable less radiation to patient, 3 ) the development of the medical image analysis software, 4) the development of the biomaterials such as those can be fabricated using RP technology with controllable strength and properties to simulate real tissues, and, perhaps the most important, 5) the computer technology: processors with a speeds exceeding $1 \mathrm{GHz}$, unlimited storage space, large random access memory (RAM), dedicated graphic cards and software for near real visualization, and internet.
It is important to recognize that the medical imaging analysis technology has been growing at a considerable pace while its application is limited by the confidence of the reconstructive surgeons. In 1990s, software algorithms that would generate 3D reconstructed images based on $2 \mathrm{D}$ slices of the CT datasets became available to the surgeon for visualizing cranio-maxillofacial deformities [7]. High-quality 3D digital models could be automatically produced without the need for manual revisions. Using an interactive program, 3D CT images can be manipulated, and osseous objects can be created to simulate the simple surgical osseous procedures [7,22]. Quantitative measurements were readily performed on the images with validated accuracy [23-33]. With this first decade of the new century, surgical simulation gained considerable progress when the separation and movement of the 3D object became available to simulate the osteotomy and the movement of the skeletal elements with 6 degrees of freedom [34-36]. Unlike the physical models of the 1990's, the computer simulation system now allowed the surgeon unlimited trials to quantify the deformity in $3 \mathrm{D}$, simulate the surgical procedure, and design the implants virtually. It becomes a useful tool for reverse engineering in clinical applications.

In parallel to the $3 \mathrm{D}$ volumetric data acquisition such as $\mathrm{CT}$, cone beam CT (CBCT), and MRI, the surface image scanners became available to the clinical practice. This surface data acquisition technology evolves from the high speed high resolution $3 \mathrm{D}$ laser scanners that acquire images without color to the $3 \mathrm{D}$ optical systems that capture the texture and color of the soft tissue envelop using synchronized multiple cameras within seconds [37]. The 3D surface image datasets can be superimposed and fused with $3 \mathrm{D}$ volumetric data such as $\mathrm{CT}$ scan data to build a more realistic digital model. These provide not only a new way to evaluate the surgical outcome without radiation exposure to the patients but also critical information for modeling and simulating the soft tissue responses to osseous movement and implant insertion. These advances have contributed to the emerging technology- the VSP system.

Today the VSP+CAD/CAM system functions as an integrated system in a new level compare to its earlier concept [38]. The medical image analysis software is capable of extracting complicated geometrical information of a skull from CT scans to build a 3D digital model virtually combining both volumetric and surface images. This 3D digital model can then be manipulated to simulate the surgical procedure such as osteotomy, and the elements repositioned for a final surgical plan that is virtually created. In addition, virtual devices (such as distraction devices and dental implants) can be integrated in the planning system. When alloplastic implants need to be customized, CAD software can be utilized to further design the implant, and then be transferred 
to a CAM system such as a stereolithograph machine to build physical models and implants. Both virtual planning and physical models provide the reconstructive surgeon with not only the individual patient specific anatomy in sufficient anatomic detail but also intra-operative guidance for repositioning and alloplastic implants when needed.

\section{A DESCRIPTION OF THE VSP+CAD/ CAM SYSTEM}

Although it may varies depending upon the specific application, a typical state of art VSP/CAD/CAM system include, but not limited to, the following components: 1) data acquisition, 2) medical image analysis, 3) 3D anthropometric analysis, 4) surgical simulation, 5) implant/template design via CAD software, 6) implant/template fabrication via $\mathrm{RP}, 7$ ) on-line communication tool, and 8) management system. Fig. 1 illustrates the $\mathrm{VSP}+\mathrm{CAD} / \mathrm{CAM}$ system established at The Craniofacial Center, University of Illinois Medical Center and Shriners Hospitals for Children-Chicago.

The input data of the VSP+CAD/CAM system includes: 1) CT scan data of a patient from either a spiral CT scanner or a cone beam CT scanner such as iCAT Next Generation 17-19 (Imaging Sciences International Inc., Hatfield, PA, USA), 2) 3D photos of

\section{Fig. 1. A VSP+CAD/CAM system}

Based upon the imported patient data that including 3D photos from a 2 port $3 \mathrm{dMDF}$ ace system (3dMD Inc.), CBCT from iCAT Next Generation 17-19 (Imaging Sciences International Inc.) and surface images from a Roland Picza laser scanner (Roland DGA Corp), a composite model is built and the surgical simulation is conducted using the virtual surgical planning software. The software is accessible from the team conference room and the operation room. The physical objects such as implant and splint can be designed virtually, and fabricated using a 3D printer, and delivered to the operation room. VSP, virtual surgical planning; CAD, computer-aided design; CAM, computer-aided manufacturing; CBCT, cone beam computed tomography.

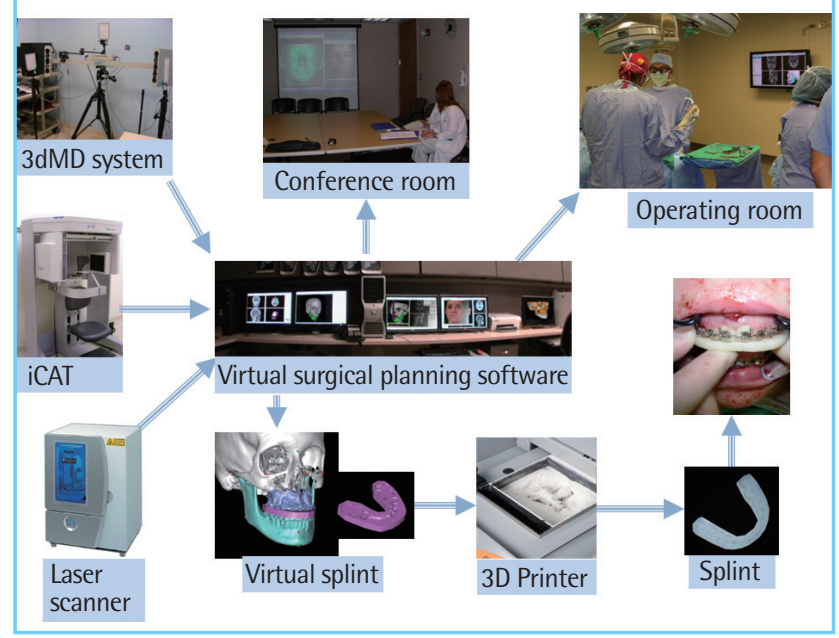

a patient from a 3D surface imaging system such as 2 port $3 \mathrm{dMD}$ Face system (3dMD Inc., Atlanta, GA, USA), and 3) 3D surface laser scans of small objects such as dental castings from a Roland Picza laser scanner (Roland DGA Corp, Irvine, CA, USA).

Both volume and surface images are processed using surgical planning software such as: 1) MIMICS (Materialise N.V., Leuven, Belgium), 2) SimPlant Pro/OMS (Materialise Dental N.V.), and 3) $3 \mathrm{dMD}$ Vultus (3dMD Inc.). The image processing includes reorientation of the CT scan data, segmentation of anatomical components (i.e., skull, mandible, soft tissue, nerve, devices), and establishment of the composite model that combines all necessary information via registration or superimposition. One of advantages of building composite model is that artifacts of metals (such as braces) in CT scan can be eliminated during the 3D modeling stage [39].

These surgical planning software allow the surgeons to conduct the user-defined 3D anthropometric analysis, which not only provide quantified information on the deformity but also interactive with surgical simulation process and provide predicted measures accordingly. They are capable to simulate surgical process such as the osteotomies of the skeletal structures, repositioning of the segments, evaluation of occlusion, 3D photo mapping, and even simulate the soft tissue response to the skeletal reconstruction. It is to be noted that the soft tissue simulation function is still in its infancy and thus has not yet been integrated into the clinical protocol.

The virtual surgical planning software can be accessed remotely in both the clinic site and the operating room via hospital-wide network. This implementation allows the surgical planning be conducted in a team conference with the attendance of surgeons, orthodontists and other clinicians in the clinic sites. This system also allows the virtual surgical plan be demonstrated in the operating room via a 42 inch monitor. Moreover, surgical planning and simulation can be conducted in the operating room when needed. This also facilitates the patient education and resident training.

Once the virtual surgical plan is approved by the surgeons, the implant and intraoperative surgical guides (such as splints for orthognathic surgery) are designed. They are further refined using CAD software and fabricated using RP techniques by manufacturers and then delivered to operating room. The communication between the surgeons and manufacturers can be conducted via online meeting.

The management of the data flow and communication is challenge and demanding. It is crucial to develop a practical protocol that all involved parties may follow. A four-week cycle protocol has been developed and applied. Following this protocol, the patient data including $\mathrm{CT}$ or $\mathrm{CBCT}, 3 \mathrm{D}$ photo, and dental cast- 
ing is collected in the first week. The VSP is conducted in the second week. So does the design of the implant and the splint. The implant and splint is fabricated and delivered in the third week and get ready for the operation in the fourth week.

\section{A CASE STUDY}

Which components of the VSP and CAD/CAM technology are critical vary with the clinical case. For this article, we illustrate a case in which this process and work flow of a VSP and CAD/ CAM approach is relevant. Fig. 2 shows the residual asymmetry of post-orthognathic surgery CT of a patient with left hemifacial microsomia. The process of VSP was used to determine whether a left repositioning zygoma would be sufficient to correct the asymmetry with autogenous reconstruction alone versus an al-

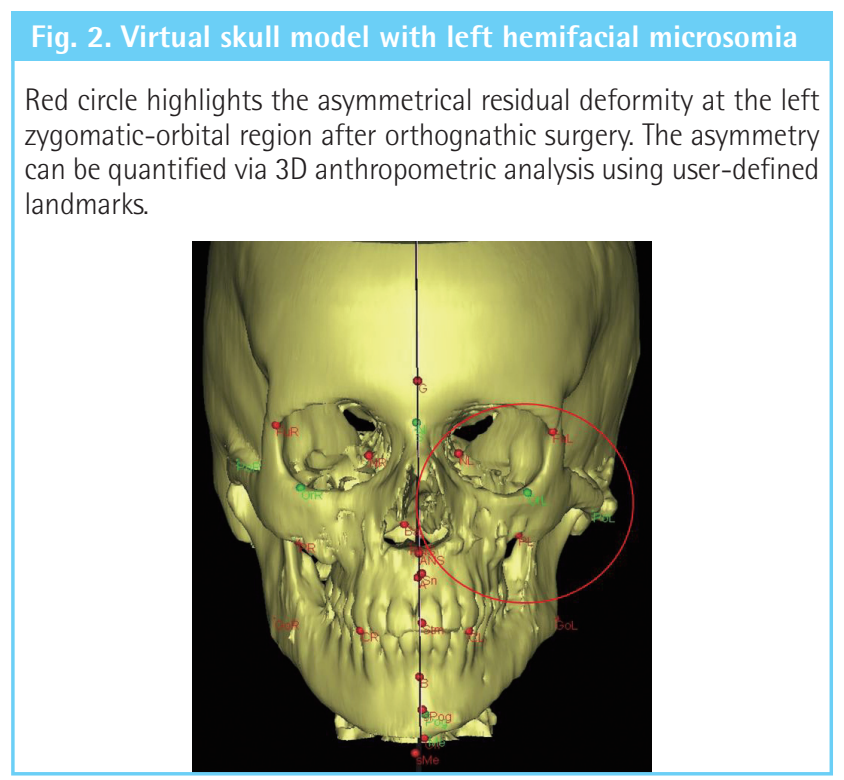

loplastic solution. The technical work flow is illustrated by Fig. 3. The CT scan of the head was acquired using a Spiral CT scanner: GE Light Speed VCT scanner (GE Medical, Milwaukee, WI, USA), with the in plane resolution or pixel size of $0.352 \times 0.352$ $\mathrm{mm}$, and the slice thickness of $2.5 \mathrm{~mm}$ (Please to be noted that

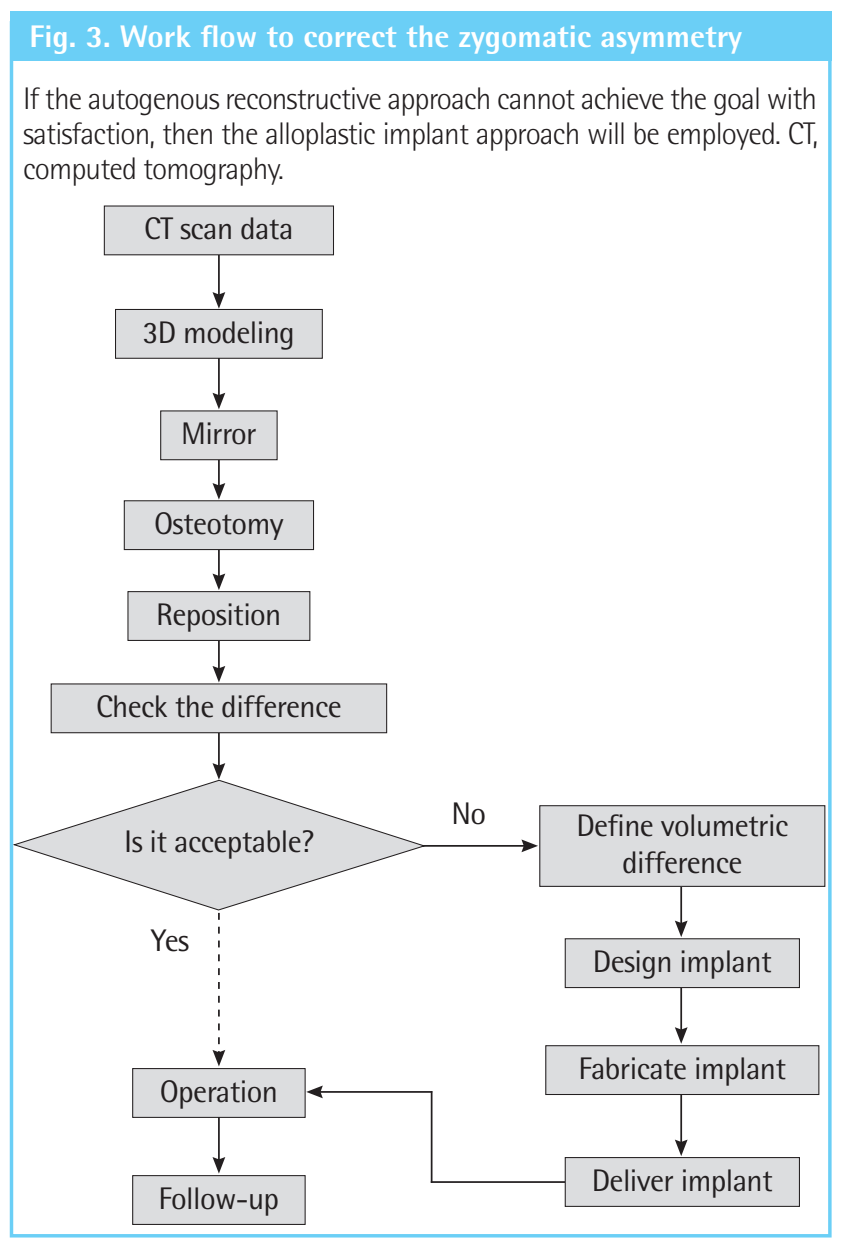

\section{Fig. 4. Mirror the unaffected side}

Mirror copy of the unaffected right side was positioned to the affected left side to achieve the symmetry. (A) Superior. (B) Inferior view.
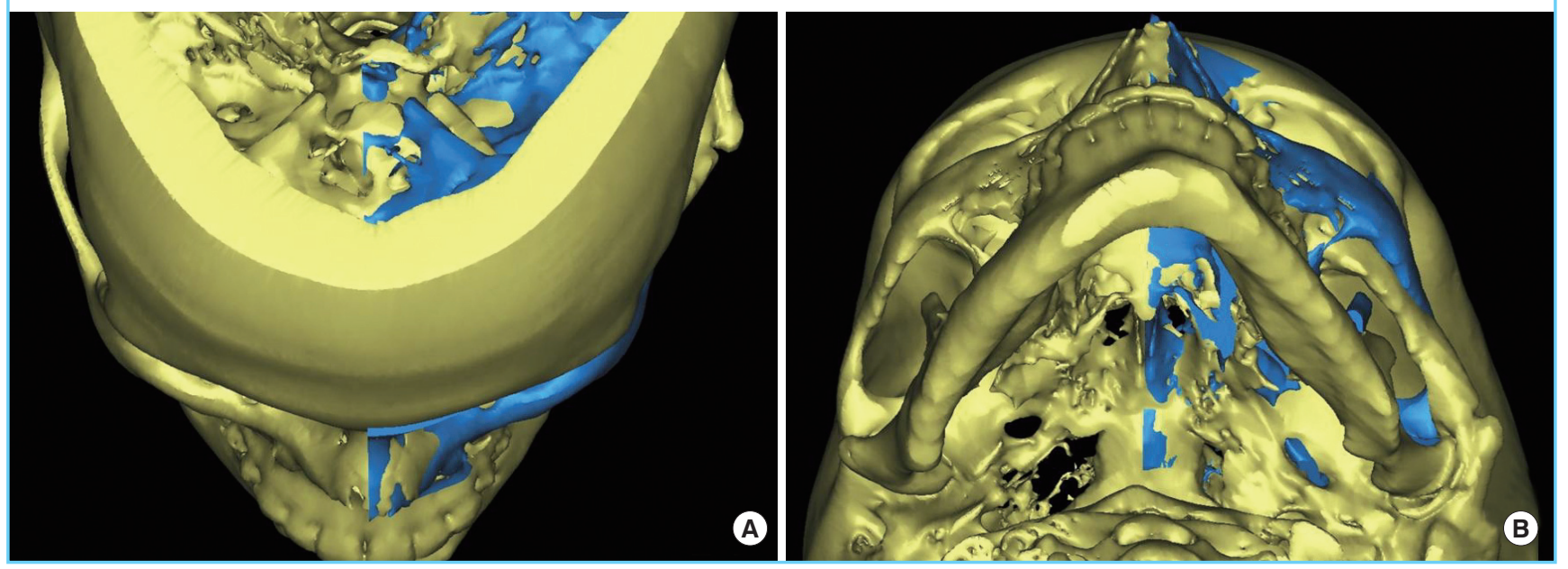
the slice thickness of no more than $1 \mathrm{~mm}$ is preferred for most applications). All data were recorded in Digital Imaging and Communications in Medicine (DICOM) format. The 3D reconstruction of the images, and the surgical simulation were performed using the MIMICS ver. 12 software (Materialise N.V.). Also, post operational 3D photos were acquired using $3 \mathrm{dMD}$ Face system and processed using $3 \mathrm{dMD}$ Patient software (3dMD Inc.).

Based on facial symmetry principle, the mirror copy of the skeletal structure was generated, as shown in Fig. 4. The midsagittal plane was initially used for the left-right mirror plane, and then further adjustment of the mirror copy was conducted to improve the overall harmony. Such adjustments were conducted using both objective criteria, that is a user-defined 3D anthropometric analysis, and subjective criteria, that is the visual observation and judgment of a surgeon and/or biomedical engineer based upon experience and professional training. The mir- ror image then set the boundary surface (the ideal surface or the reference surface) against which various surgical options were assessed.

The treatment plan was simulated with two different approaches to achieving symmetry: autogenous skeletal reconstruction versus alloplastic implant. Autogenous reconstruction is generally the favored approach because of long term stability and without the long term concern of alloplastic tissue interface problems. Thus the surgeon must decide if an autogenous reconstruction can achieve the desired outcome. By performing the surgery virtually, the surgeon can optimize the various surgical options and the patient has an opportunity to visualize the complexity of achieving the desired result.

The prediction from the autogenous skeletal reconstruction approach is presented in Fig. 5. Various designs of the osteotomy and reposition could not achieve the tolerance limit for asym-

\section{Fig. 5. The reconstructive approach}

The bony segment in red was separated (A) and moved to fit to the mirror reference in blue and the difference in all three reposition scenario (B-D) was not acceptable to the tolerance limit.
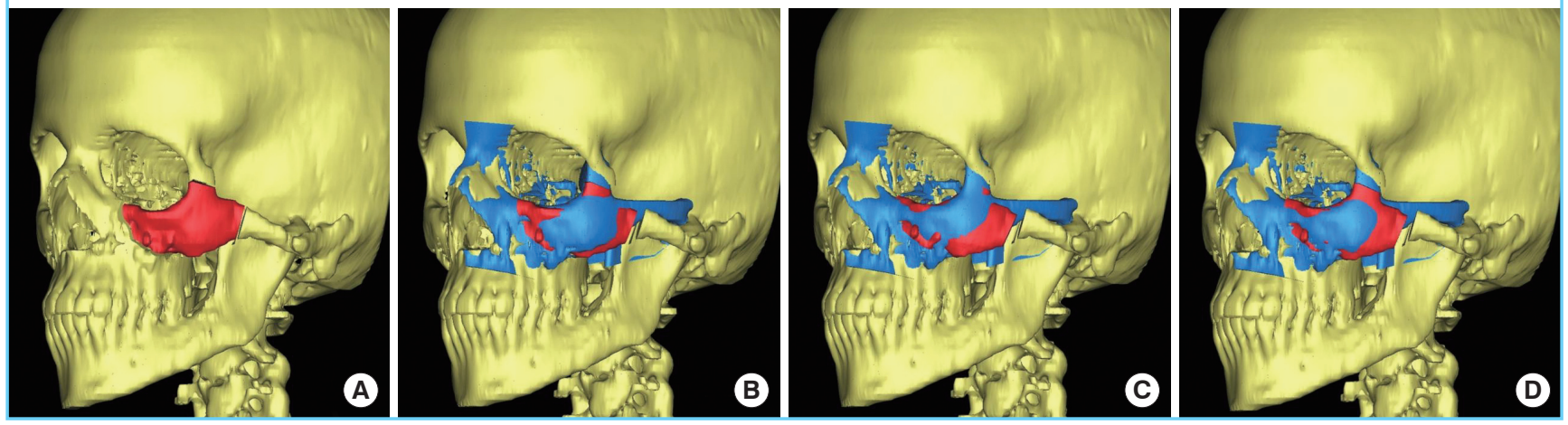

\section{Fig. 6. The alloplastic implant approach}

The volumes in purple and in light green predict the implant to achieve the symmetry. (A) Frontal, (B) oblique, (C) left view. The volume in light green is thin.
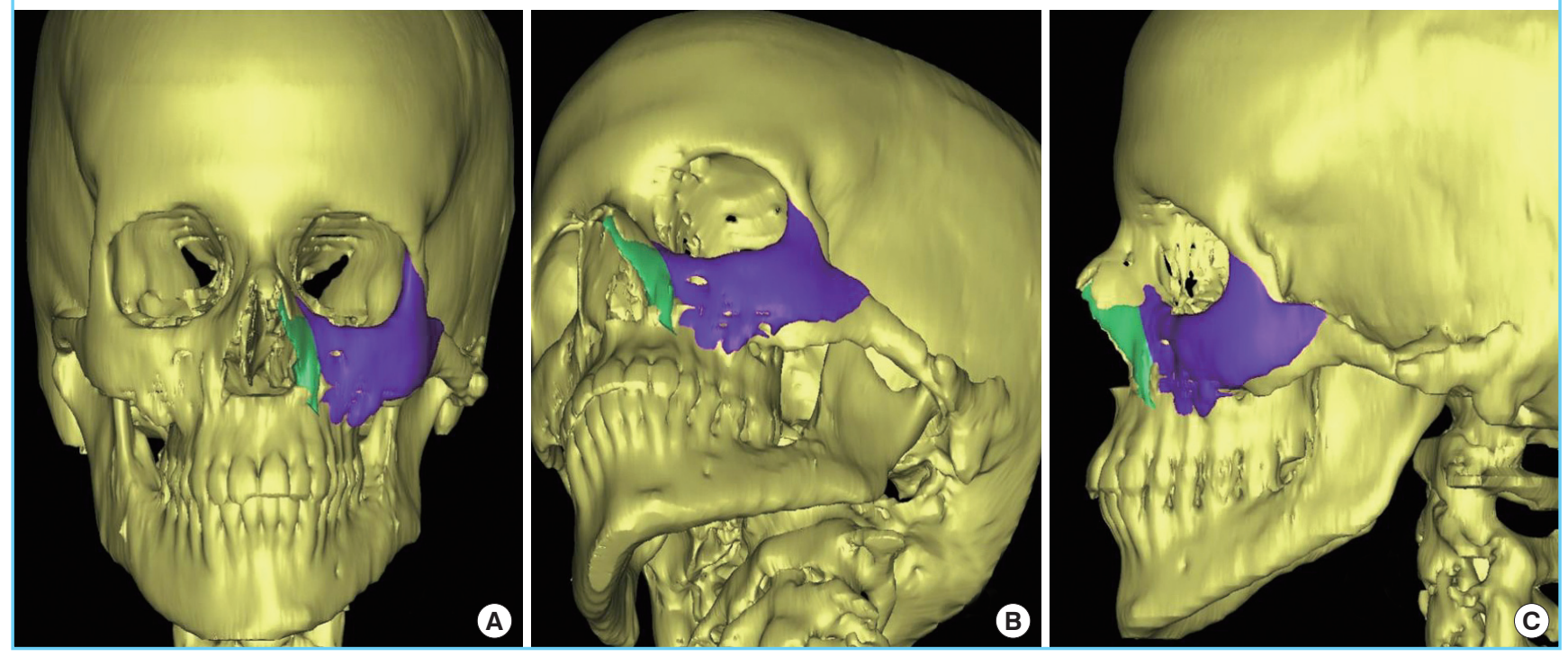


\section{Fig. 7. Implant and skull model fabricated using RP technology}

(A) The plaster piece in light blue is the initial design for surgeon to review. Surgeon's modification is marked on the skull model using pencil. (B) The final design of the custom HTR-PMI implant, which was fabricated with porous PMMA material (Biomed Microfixation).
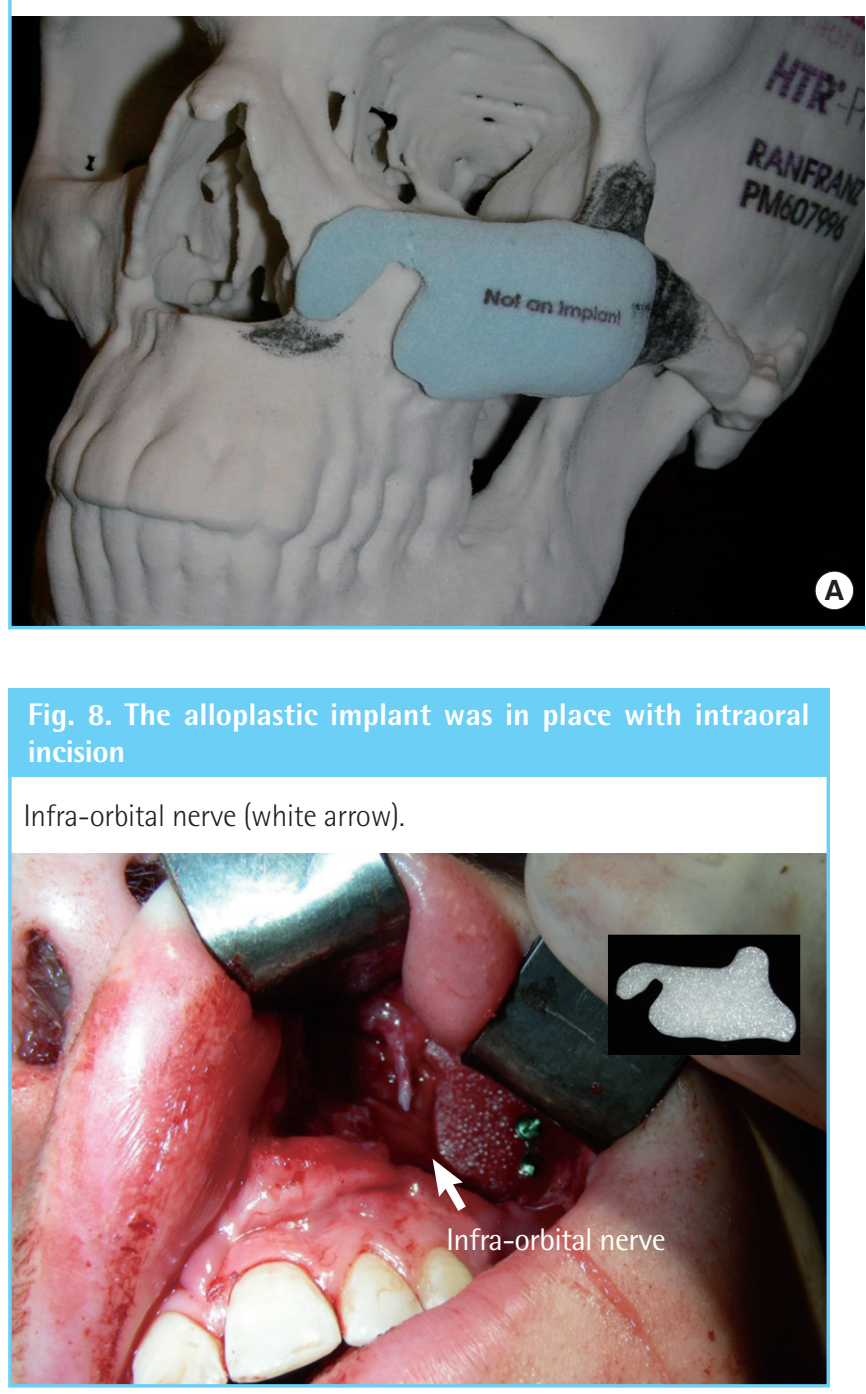

metry by the patient and the surgeon. Thus a custom alloplastic implant was designed that would achieve the desired symmetry (Fig. 6).

For the alloplastic implant approach, Boolean operation (on polygons) [40] was used to calculate the differences between the actual skeletal surface and the reference surface, and then initial design of the implant was extracted accordingly. In such initial design there were thin edges, such as in the nasal side, which were unfavorable to the manufacture process. The implant was thus redesigned by the manufacturer. From surgeon point of view, however, the redesigned piece was too small and might be unstable in long term, additional extension to both lateral orbital rim and zygomatic process were needed. Further modifications were made in the final design of the alloplastic implant, as shown in Fig. 7.

A plaster skull model and a plaster prototype of the implant

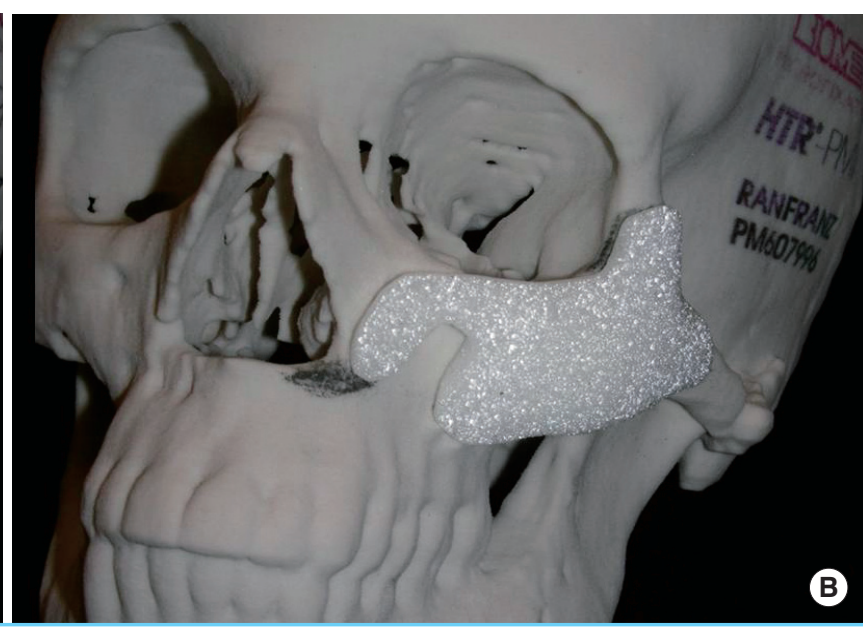

were made by RP facility and delivered to the surgeon for communication and approval, as shown in Fig. 7. The HTR-PMI implant with the final design was made using porous PMMA material (Biomed Microfixtion, Jacksonville, FL, USA), sterilized and delivered to the operation room directly. Also delivered to the operation room was the surgical plan in the software.

After the skeletal surface was exposed, the implant fitted well to the surface topography and was then fixed with titanium screws, as shown in Fig. 8. Intraoperative exposure of the osseous defects was facilitated by on-site inspection of the plaster skull model. No unexpected deformities or untoward injuries were encountered during the operation. The fit of the HTR-PMI implant was extremely well, and consequently, no adjustments were needed. The patient was followed for 2 years and the facial symmetry was achieved. There were no complications.

\section{CONCLUSIONS}

By performing the surgery virtually, the surgeon can compare and optimize the various surgical options and the patient has an opportunity to visualize the complexity of achieving the desired result. By deliver the individual implant and splint to the operation room directly, the virtual surgical plan can be transferred to the operating table instead of just stay in the computer. A less invasive surgical procedure, less time-consuming and adequate aesthetic results can be achieved. Many patients have been benefitted from the VSP+CAD/CAM system.

\section{ACIKNOWLED GEMENT}

Informed consent has been obtained for the publication of the 
photos in this article. The VSP + CAD/CAM system presented in this article is partially supported by Face the Future Foundation, and Shriners Hospitals for Children.

\section{REFERENCES}

1. Hounsfield GN. Computerized transverse axial scanning (tomography). 1. Description of system. Br J Radiol 1973; 46:1016-22.

2. Ambrose J. Computerized transverse axial scanning (tomography). 2. Clinical application. Br J Radiol 1973;46:1023-47.

3. Eufinger H, Wehmoller M, Harders A, et al. Prefabricated prostheses for the reconstruction of skull defects. Int J Oral Maxillofac Surg 1995;24:104-10.

4. Metzger MC, Hohlweg-Majert B, Schwarz U, et al. Manufacturing splints for orthognathic surgery using a three-dimensional printer. Oral Surg Oral Med Oral Pathol Oral Radiol Endod 2008;105:e1-7.

5. Vannier MW, Marsh JL, Warren JO. Three dimensional CT reconstruction images for craniofacial surgical planning and evaluation. Radiology 1984;150:179-84.

6. Toth BA, Ellis DS, Stewart WB. Computer-designed prostheses for orbitocranial reconstruction. Plast Reconstr Surg 1988;81:315-24.

7. Robb RA. Biomedical imaging, visualization, and analysis. New York: Wiley-Liss; 2000.

8. Woolson ST, Dev P, Fellingham LL, et al. Three-dimensional imaging of bone from computerized tomography. Clin Orthop Relat Res 1986;(202):239-48.

9. Solar P, Ulm C, Lill W, et al. Precision of three-dimensional CT-assisted model production in the maxillofacial area. Eur Radiol 1992;2:473-7.

10. Eufinger $\mathrm{H}$, Wehmoller M, Machtens E, et al. Reconstruction of craniofacial bone defects with individual alloplastic implants based on CAD/CAM-manipulated CT-data.J Craniomaxillofac Surg 1995;23:175-81.

11. Wehmoller M, Eufinger $\mathrm{H}$, Kruse $\mathrm{D}$, et al. CAD by processing of computed tomography data and CAM of individually designed prostheses. Int J Oral Maxillofac Surg 1995;24:90-7.

12. Klein HM, Schneider W, Alzen G, et al. Pediatric craniofacial surgery: comparison of milling and stereolithography for 3D model manufacturing. Pediatr Radiol 1992;22:458-60.

13. Housholder R. Molding process. US Patent 4,247,508. Filed December 3, 1979. Published January 27, 1981.

14. Hull CW. Apparatus for production of three-dimensional objects by stereolithography. US Patent 4,575,330. Filed Aug 8, 1984. Published March 11, 1986.

15. Deckard C. Method and apparatus for producing parts by selective sintering. U.S. Patent 4,863,538, Filed October 17, 1986. Published September 5, 1989.

16. McGurk M, Amis AA, Potamianos P, et al. Rapid prototyping techniques for anatomical modelling in medicine. Ann R Coll Surg Engl 1997;79:169-74.

17. Sailer HF, Haers PE, Zollikofer CP, et al. The value of stereolithographic models for preoperative diagnosis of craniofacial deformities and planning of surgical corrections. Int J Oral Maxillofac Surg 1998;27:327-33.

18. Santler G, Karcher H, Ruda C. Indications and limitations of three-dimensional models in cranio-maxillofacial surgery. J Craniomaxillofac Surg 1998;26:11-6.

19. Joffe JM, McDermott PJ, Linney AD, et al. Computer-generated titanium cranioplasty: report of a new technique for repairing skull defects. Br J Neurosurg 1992;6:343-50.

20. Binder WJ, Kaye A. Reconstruction of posttraumatic and congenital facial deformities with three-dimensional computer-assisted custom-designed implants. Plast Reconstr Surg 1994;94:775-85.

21. Tanaka Y, Matsumoto K, Song S, et al. Reconstruction of a cranial bone defect with hydroxyapatite and free flap transfer. J Craniofac Surg 1997;8:141-5.

22. Fukuta F, Jackson IT, McEwan CM, et al. Three- dimensional imaging in craniofacial surgery: a review of the role mirror image production. Eur J Plast Surg 1990;13:209-17.

23. Altobelli DE, Kikinis R, Mulliken JB, et al. Computer-assisted three-dimensional planning in craniofacial surgery. Plast Reconstr Surg 1993;92:576-85.

24. Hildebolt CF, Vannier MW. Three-dimensional measurement accuracy of skull surface landmarks. Am J Phys Anthropol 1988;76:497-503.

25. Hildebolt CF, Vannier MW, Knapp RH. Validation study of skull three-dimensional computerized tomography measurements. Am J Phys Anthropol 1990;82:283-94.

26. Lo LJ, Marsh JL, Vannier MW, et al. Craniofacial computerassisted surgical planning and simulation. Clin Plast Surg 1994;21:501-16.

27. Cavalcanti MG, Vannier MW. Quantitative analysis of spiral computed tomography for craniofacial clinical applications. Dentomaxillofac Radiol 1998;27:344-50.

28. Lo LJ, Lin WY, Wong HF, et al. Quantitative measurement on three-dimensional computed tomography: an experimental validation using phantom objects. Chang Gung Med J 2000;23:354-9.

29. Cavalcanti MG, Rocha SS, Vannier MW. Craniofacial measurements based on 3D-CT volume rendering: implications for clinical applications. Dentomaxillofac Radiol 2004;33: 170-6. 
30. Lopes PM, Moreira CR, Perrella A, et al. 3-D volume rendering maxillofacial analysis of angular measurements by multislice CT. Oral Surg Oral Med Oral Pathol Oral Radiol Endod 2008;105:224-30.

31. Moreira CR, Sales MA, Lopes PM, et al. Assessment of linear and angular measurements on three-dimensional conebeam computed tomographic images. Oral Surg Oral Med Oral Pathol Oral Radiol Endod 2009; 108:430-6.

32. Berco M, Rigali PH Jr, Miner RM, et al. Accuracy and reliability of linear cephalometric measurements from conebeam computed tomography scans of a dry human skull. Am J Orthod Dentofacial Orthop 2009;136:17.e1-9.

33. Fleming PS, Marinho V, Johal A. Orthodontic measurements on digital study models compared with plaster models: a systematic review. Orthod Craniofac Res 2011;14:1-16.

34. Cynthia DB, Steven S, Anil M, et al. A survey of interactive mesh-cutting techniques and a new method for implementing generalized interactive mesh cutting using virtual tools. J
Visual Comput Animat 2002;13:21-42.

35. Zachow S, Gladilina E, Saderb R, et al. Draw and cut: intuitive $3 \mathrm{D}$ osteotomy planning on polygonal bone models. Int Congr Ser 2003; 1256:362-9.

36. Westermark A, Zachow S, Eppley BL. Three-dimensional osteotomy planning in maxillofacial surgery including soft tissue prediction. J Craniofac Surg 2005; 16:100-4.

37. Lane C, Harrell W Jr. Completing the 3-dimensional picture. Am J Orthod Dentofacial Orthop 2008;133:612-20.

38. Chua CK, Chou SM, Ng WS, et al. An integrated experimental approach to link a laser digitiser, a CAD/CAM system and a rapid prototyping system for biomedical applications. Int J Adv Manuf Technol 1998; 14:110-5.

39. Xia JJ, Gateno J, Teichgraeber JF. Three-dimensional computer-aided surgical simulation for maxillofacial surgery. Atlas Oral Maxillofac Surg Clin North Am 2005;13:25-39.

40. MIMICS version 12 reference guide. Leuven (BE): Materialise N.V.; 2009. 